\title{
Estudio retrospectivo de la ocurrencia de accidente cerebrovascular en pacientes diabéticos.
}

\author{
NEIRA SANCHEZ Elsa Rosa, SECLEN SANTISTEBAN Segundo *
}

\section{SUMMARY}

Objective: To determine diabetes mellitus frequency in hospitalized patients with stroke diagnosis, to describe the clinical characteristics of stroke in diabetic and no diabetic patients and identify other risk factors for stroke in diabetic patients. Material and Methods: It's a retrospective study that includes hospitalized patients with discharge of stroke, selection judgements were applied to define stroke and diabetes mellitus. Results: The prevalence of ischemic stroke was greater in diabetic patients that in no diabetics $(83 \%$ vs. $57 \%, p<0.005)$. From diabetic patients, 11 were diagnosed at the hospitalization moment, from 30 patients with previous diagnosis of diabetes, $33 \%$ had 10 to 15 years of diagnosis and $24 \%$ had more of 15 years of disease. The majority of diabetic patients were carriers of diabetic nephropathy $(73 \%)$ and diabetic retinopathy (46\%). Hypertension was 1.2 times more frequent en diabetic patients that in no diabetics. Also we found more disturbances in the lipid profile of diabetic patients. Conclusions: $15 \%$ of the patients with stroke had diagnosis of Diabetes Mellitus. Ischemic stroke was more frequent in diabetic patients. In diabetic patients, stroke is more frequent in those who have more that ten years of disease, in which diabetes contributes to increase the vascular damage, but also they present other pathologies that contribute to this damage, like hypertension, cardiovascular disease and lipid disorders. ( Rev Med Hered 2000; 11:5461 ).

KEY WORDS: Diabetes Mellitus, stroke, ischemic stroke.

\section{RESUMEN}

Objetivo: Determinar la frecuencia de diabetes mellitus en la población hospitalizada por ACV, describir las características clínicas del ACV en pacientes diabéticos y no diabéticos y determinar la presencia de otros factores de riesgo para ACV en pacientes diabéticos. Material y Métodos: Es un estudio retrospectivo que incluye pacientes hospitalizados con diagnóstico al alta de ACV, a los que se aplicaron criterios de selección para definir ACV y Diabetes Mellitus. Resultados: La prevalencia del ACV tipo isquémico fue mayor en los pacientes diabéticos que en los no diabéticos ( $83 \%$ vs. $57 \%$, p <0.005). De los pacientes diabéticos, 11 de ellos fueron diagnosticados en el momento de la hospitalización, de los 30 pacientes con diagnóstico previo de esta patología el 33\% tenían de 10 a 15 años de diagnóstico de diabetes y $24 \%$ tenían más de 15 años de enfermedad. La mayoría de pacientes diabéticos eran portadores de nefropatía diabética $(73 \%)$ y retinopatía diabética $(46 \%)$. La hipertensión arterial fue 1.2 veces más frecuente en los pacientes diabéticos que en los no diabéticos. También se encontraron más alteraciones en el perfil 
lipídico de los pacientes diabéticos. Conclusiones: El 15\% de la población con ACV tuvo diagnóstico de Diabetes Mellitus. El ACV tipo isquémico fue mas frecuente en los pacientes diabéticos. En los pacientes diabéticos el ACV ocurre mas frecuentemente en aquellos con mas de diez años de enfermedad en los que la diabetes contribuye a aumentar el daño vascular, pero además presentan otras patologías que contribuyen a este daño, como hipertensión arterial, enfermedad cardiovascular y dislipidemias. ( Rev Med Hered 2000; 11:54-61).

PALABRAS CLAVE: Diabetes Mellitus, accidente cerebrovascular, accidente cerebrovascular isquémico.

\section{INTRODUCCION}

La diabetes mellitus es una enfermedad que se produce como consecuencia de defectos en la secreción y/ o acción de la insulina y que producen un conjunto de trastornos metabólicos caracterizados por hiperglicemia. La hiperglicemia crónica de la diabetes está asociada a complicaciones a largo plazo, disfunción y falla de diversos órganos que incluyen retinopatía, neuropatía periférica y neuropatía autonómica. Asimismo, los pacientes con diabetes tienen una incidencia aumentada de enfermedad ateroesclerótica cardiovascular, vascular periférica y enfermedad cerebrovascular.

Diversos estudios han demostrado una alta prevalencia de accidente cerebrovascular (ACV) en la población diabética, con diabetes no diagnosticada y con intolerancia a la glucosa, enfatizándose que los sujetos con diabetes tienen mayor riesgo de accidente cerebrovascular (1) y mayor morbimortalidad. A pesar que la asociación entre diabetes y accidente cerebrovascular puede ocurrir a través de diversos factores de riesgo, varios estudios han observado una asociación independiente de la diabetes con un riesgo elevado de accidente cerebrovascular isquémico y de todo tipo, tanto para hombres como para mujeres $(2,3)$. La diabetes mellitus ha sido descrita como un factor de riesgo para el accidente cerebrovascular isquémico, pero no para el hemorrágico, y se sabe también que la frecuencia de accidente isquémico transitorio (AIT) no está aumentada en los pacientes con diabetes comparada con la población general y aparentemente, la diabetes tampoco se asociaría con un mayor tamaño del infarto cerebral (4).

El presente estudio describe las características clínicas del accidente cerebrovascular como complicación de la diabetes mellitus tipo 2 e identifica la influencia de otros factores de riesgo en la ocurrencia del accidente cerebrovascular en la población diabética como una contribución al estudio de esta patología en nuestro medio.

\section{MATERIAL Y METODOS}

El presente es un estudio descriptivo y retrospectivo que incluye a 270 pacientes hospitalizados en el Hospital Nacional Cayetano Heredia cuyo diagnóstico al alta fue accidente cerebrovascular entre los años 1990 a 1996.

\section{Criterios de Selección:}

Se definió accidente cerebrovascular basándose en los hallazgos clínicos convencionales de presentación y evolución neurológica. Los diagnósticos fueron confirmados con estudios tomográficos y/o arteriográficos durante la hospitalización y/o en la evolución en el consultorio externo de Neurología, así como los hallazgos quirúrgicos de los pacientes sometidos a cirugía.

Para definir a los pacientes diabéticos se aplicaron los actuales criterios de diagnóstico, confirmándose el diagnóstico en el transcurso de la hospitalización y durante el seguimiento de los pacientes en consulta externa. Las complicaciones crónicas de la diabetes mellitus se definieron según criterios establecidos en la bibliografía (5).

La población fue dividida en dos grupos: Pacientes con ACV y diagnóstico de diabetes (41 pacientes) y pacientes con ACV y sin evidencia de diabetes (229 pacientes).

La comparación de estos dos grupos sirvió para determinar la influencia de la diabetes en la ocurrencia del accidente cerebrovascular, así como la presencia en la población diabética de los diferentes factores de riesgo para la ocurrencia de ACV.

\section{Análisis Estadístico:}

Se utilizó, el paquete estadístico EPI INFO. Se realizaron las pruebas de chi-cuadrado, $\mathrm{t}$ de student $\mathrm{y}$ la prueba $\mathrm{z}$ asumiéndose un nivel de significación de $\mathrm{p}<0.05$.

\section{RESULTADOS}

En la población total de 270 pacientes se encontró que $41(15 \%)$ eran portadores de diabetes mellitus tipo 
2 y ninguno era portador de diabetes mellitus tipo 1 .

Tipos de ACV

La distribución fue de 164 pacientes (61\%) con ACV tipo isquémico, 79 (29\%) con ACV tipo hemorrágico, $16(6 \%)$ con Accidente Isquémico Transitorio (AIT) y $11(4 \%)$ con Hemorragia Subaracnoidea (HSA). Analizando la distribución de los diferentes tipos de ACV en los pacientes diabéticos y no diabéticos, se encontró, que la prevalencia del ACV tipo isquémico fue significativamente mayor en los pacientes diabéticos que en los no diabéticos ( $83 \%$ vs. $57 \%$, p <0.005), mientras que el ACV tipo hemorrágico fue significativamente mas frecuente en los pacientes no diabéticos que en los diabéticos ( $32 \%$ vs $12 \%$, p <0.005). Los otros tipos de ACV fueron menos frecuentes, el AIT se presentó en $5 \%$ de los pacientes diabéticos y en $6 \%$ de los no diabéticos y la HSA sólo se observó en $5 \%$ de los pacientes no diabéticos (Gráfico $\mathrm{N}^{\circ} 1$ ).

\section{Territorios cerebrales afectados y extensión del ACV}

La mayor parte de accidentes cerebrovasculares afectaron la arteria cerebral media, en proporción similar en pacientes diabéticos (69\%) como en no diabéticos (64\%). Otro hallazgo importante fue el tamaño de los territorios afectados; se encontró que los accidentes cerebrovasculares que afectan más del $30 \%$ del parénquima cerebral de ambos hemisferios cerebrales fueron mas frecuentes en los pacientes diabéticos que en los no diabéticos $(46 \%$ vs. $30 \%$, p<0.025).

\section{Género, edad y raza}

En el género no se observó preponderancia en ninguno de los grupos. La relación hombre/mujer fue de 1 en

\section{Gráfico $\mathrm{N}^{\circ} 1$. Distribución de los tipos de ACV en pacientes diabéticos $(n=41)$ y no diabéticos $(n=229)$.}

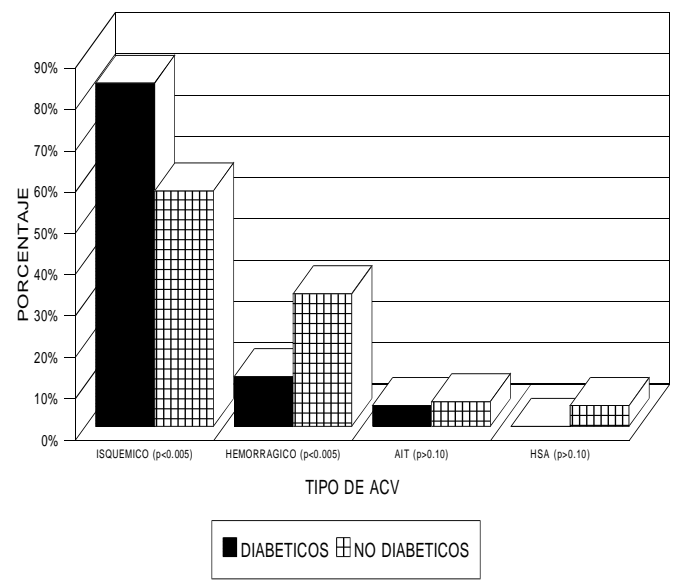

los pacientes diabéticos y 1.3 en los pacientes no diabéticos. La edad promedio de presentación del primer ACV en diabéticos y no diabéticos fue similar, 60.9 años y 59.8 años, respectivamente. Es importante señalar, sin embargo que la presentación en los diabéticos hombres fue a edad significativamente mas joven que en las diabéticas mujeres (56.2vs. $65.5 \mathrm{p}<0.005)$, esta diferencia no se observó en los pacientes no diabéticos (58.5 vs. 61.6). El mayor porcentaje de los pacientes en ambos grupos fue de raza mestiza (93.4\%).

\section{Ocurrencia de ACV y tiempo de duración de la diabetes}

De los 41 pacientes con diagnóstico de diabetes, 30 pacientes ingresaron con diagnóstico previo de esta patología y en ellos la edad promedio de diagnóstico fue 54.3 años; en los once pacientes restantes, el diagnóstico de diabetes fue hecho recién en el momento de la hospitalización.

La distribución del tiempo de enfermedad de diabetes en los 30 pacientes con diagnóstico de ACV se muestra en el gráfico $\mathrm{N}^{\circ} 2$, en el cual se evidencia que $33 \%$ de las pacientes tenían de 10 a 15 años de diagnóstico de diabetes mellitus tipo 2 y $24 \%$ tenían mas de 15 años de diagnóstico.

Tiempo de enfermedad de diabetes y presencia de otras patologías relacionada con la ocurrencia de ACV

Como se muestra en el gráfico $\mathrm{N}^{\circ} 2$, se encontró una correlación directa entre la ocurrencia de ACV y el tiempo de enfermedad de diabetes. Esta tendencia también se observa al analizar la ocurrencia de ACV y su relación con la hipertensión arterial, la nefropatía diabética y la retinopatía diabética, encontrándose que al aumentar el tiempo de enfermedad de diabetes aumenta la ocurrencia

Gráfico $\mathrm{N}^{\circ} 2$. Ocurrencia de ACV en relación al tiempo de duración de la diabetes y la presencia de otras complicaciones $(n=30)$.

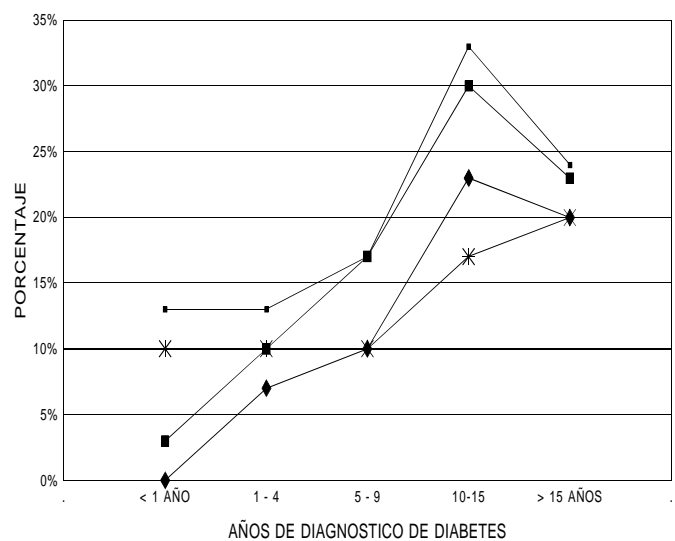

- OCURRENCIA DE ACV * HIPERTENSION ARTERIAL NEFROPATIA DIABETICA RETINOPATIA DIABETICA 


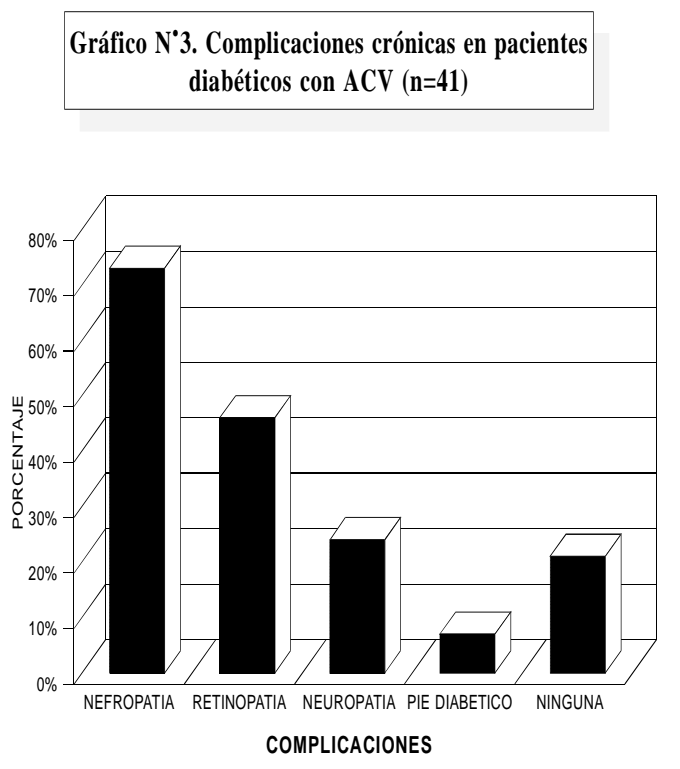

de estas patologías. El grupo de pacientes a los que se les diagnosticó la diabetes durante la hospitalización, tenía una alta frecuencia de otras patologías asociadas como hipertensión arterial (73\%) y nefropatía diabética (45\%).

\section{Complicaciones crónicas de la diabetes asociada con la ocurrencia del ACV}

Los pacientes diabéticos en los que ocurrió ACV eran portadores en su mayoría de nefropatía diabética (73\%) seguidos por 19 pacientes portadores de retinopatía diabética (46\%). Las complicaciones menos significativas fueron pie diabético y neuropatía diabética. Nueve pacientes no presentaron ningún tipo de complicación crónica de la diabetes en el momento del ACV (Gráfico N³).

\section{Desórdenes cardiovasculares}

El diagnóstico previo de hipertensi6n arterial fue el antecedente cardiovascular más frecuente, 143 pacientes $(53 \%)$ de la población total tenían este antecedente. Al comparar los grupos se encontró que los pacientes diabéticos tenían una mayor prevalencia de hipertensión arterial que los no diabéticos ( $68 \%$ vs. $61 \%, \mathrm{p}<0.025)$. La fibrilación auricular fue otro trastorno cardiovascular importante; $20 \%$ de los pacientes diabéticos y $15 \%$ de los pacientes no diabéticos eran portadores de fibrilación auricular.

El antecedente de infarto agudo de miocardio fue mas prevalente en la población diabética (12\%) que en la nodiabética $(4 \%)(\mathrm{p}<0.025)$. Lo mismo ocurrió con el antecedente de insuficiencia cardíaca congestiva, la cual fue mas frecuente en los pacientes diabéticos que en los no diabéticos (20\% vs. $6 \%, \mathrm{p} \sim 0.005)$. Só1o un
Tabla $\mathrm{N}^{\circ} 1$. Transtornos cardiovasculares en pacientes con diagnóstico de ACV.

\begin{tabular}{lccc} 
& DIABETICOS & NO DIABETICOS & $p$ \\
\hline Diagnóstico previo de & & & \\
Hipertensión arterial & $28(68 \%)$ & $115(61 \%)$ & $<0.025$ \\
Fibrilación auricular & $8(20 \%)$ & $34(15 \%)$ & NS \\
Insuficiencia cardiaca & $8(20 \%)$ & $14(6 \%)$ & $<0.005$ \\
Infarto agudo de miocardio & $5(12 \%)$ & $9(4 \%)$ & $<0.025$ \\
Valvulopatía & $1(2 \%)$ & $10(4 \%)$ & NS \\
\hline
\end{tabular}

paciente diabético era portador de valvulopatía (Tabla $\mathrm{N}^{\circ} 1$ ). Además se encontró que un número significativo de pacientes $(60 \%)$ era portador de hipertrofia ventricular izquierda.

\section{Antecedente de ACV previo}

$\mathrm{El}$ antecedente de accidente cerebrovascular fue mas frecuente en los pacientes diabéticos que en los no diabéticos. La frecuencia en los pacientes diabéticos fue $27 \%$ y en los no diabéticos fue de $15 \%(\mathrm{p}<0.05)$.

\section{Perfil lipídico}

Se estudió el perfil lipídico en 21 pacientes diabéticos y 95 pacientes no diabéticos. Se encontró diferencia significativa en los valores de colesterol total mayores de $200 \mathrm{mg} / \mathrm{dl}$ entre los pacientes diabéticos (67\%) y los pacientes no diabéticos $(47 \%)$ ( $\mathrm{p}<0.05)$, en los valores de colesterol LDL mayores de $130 \mathrm{mg} / \mathrm{dl}$ en pacientes diabéticos $(71 \%)$ comparados con los no diabéticos $(53 \%)(\mathrm{p}<0.025)$ y finalmente en los valores de colesterol HDL menores de $40 \mathrm{mg} / \mathrm{dl}$ en pacientes diabéticos (76\%) comparado con los pacientes no diabéticos $(67 \%)$ $(\mathrm{p}<0.05)$. No se encontró diferencia significativa en los niveles de triglicéridos (Tabla $\mathrm{N}^{\circ} 2$ ).

\section{Eventos durante la hospitalización}

El promedio de días de hospitalización fue similar en los pacientes diabéticos (14.7 días) y en los no diabéticos (14.6 días). Otra variable que se analizó fue, las complicaciones que se dieron durante la hospitalización (Tabla $\mathrm{N}^{\circ} 3$ ). La insuficiencia cardiaca fue la complicación mas frecuente tanto en los pacientes diabéticos (17\%) que en los no diabéticos (7\%) 
Tabla $\mathrm{N}^{\circ} 2$. Perfil lipídico en pacientes diabéticos y no diabéticos con ACV.

\begin{tabular}{lccc} 
& DIABETICOS & NO DIABETICOS & $P$ \\
\hline Colesterol Total $>200 \mathrm{mg} / \mathrm{dl}$ & $14(67 \%)$ & $45(47 \%)$ & $<0.05$ \\
Colesterol LDL $>130 \mathrm{mg} / \mathrm{dl}$ & $15(71 \%)$ & $50(53 \%)$ & $<0.025$ \\
Colesterol HDL $<40 \mathrm{mg} / \mathrm{dl}$ & $17(76 \%)$ & $59(67 \%)$ & $<0.05$ \\
Triglicéridos $>150 \mathrm{mg} / \mathrm{dl}$ & $3(14 \%)$ & $14(15 \%)$ & NS \\
\hline
\end{tabular}

$(\mathrm{p}<0.025)$; la infección del tracto urinario fue también más frecuente en los diabéticos (13\%) que en los no diabéticos $(3 \%)(\mathrm{p}<0.005)$ y un paciente diabético (2\%) presentó infarto agudo del miocardio durante su hospitalización.

\section{Mortalidad}

La mortalidad en la población total hospitalizada con diagnóstico de ACV fue de $1.9 \%$, fallecieron cinco pacientes de la población total de 270 pacientes hospitalizados, los cinco pacientes eran no diabéticos y se les diagnosticó como portadores de ACV tipo hemorrágico. Cuatro de ellos fallecieron por falla ventilatoria secundaria a enclavamiento y uno de ellos por sepsis y shock séptico.

\section{DISCUSION}

En la literatura se señala que entre el 2 y $7 \%$ de los pacientes que sufren accidente cerebrovascular son portadores de diabetes mellitus tipo $2(6,7)$. En el presente estudio se encontró que $15 \%$ de la población hospitalizada con ACV era a su vez diabética. Este alto porcentaje se puede explicar por la alta prevalencia de la diabetes en nuestro medio y por el hecho que el Hospital Nacional Cayetano Heredia cuenta con un programa especial para pacientes diabéticos, lo que favorece la captación de pacientes con esta patología.

Los estudios en pacientes diabéticos reportan que el $\mathrm{ACV}$ tipo isquémico es mas frecuente en hombres y mujeres diabéticas y que hay una menor prevalencia de ACV tipo hemorrágico y Hemorragia Subaracnoidea (HSA) $(1,7,8)$. En el presente estudio hemos corroborado que el tipo de ACV más frecuente en la población

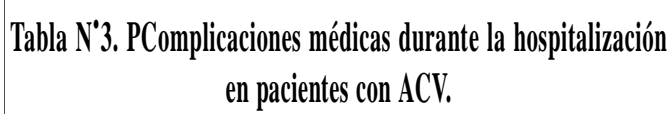

\begin{tabular}{lccc} 
& DIABETICOS & NO DIABETICOS & $P$ \\
\hline Insuficiencia cardiaca mgld & $7(17 \%)$ & $16(7 \%)$ & $<0.025$ \\
Infección del tracto urinario & $5(13 \%)$ & $7(3 \%)$ & $<0.005$ \\
Infarto agudo de miocardio & $1(2 \%)$ & $0(0 \%)$ & $<0.005$ \\
\hline
\end{tabular}

diabética es el de tipo isquémico (83\%) y que al mismo tiempo este es más frecuente en los pacientes diabéticos que en los no diabéticos; sólo $12 \%$ de los pacientes diabéticos presentaron $\mathrm{ACV}$ tipo hemorrágico y ningún paciente diabético presentó HSA. El ACV tipo hemorrágico fue mas frecuente en los pacientes no diabéticos que en los diabéticos y no se encontró diferencia significativa en la ocurrencia de Accidente isquémico Transitorio (AIT).

El territorio cerebral mas afectado por el ACV en pacientes diabéticos y no diabéticos fue el de la arteria cerebral media, seguido en frecuencia por los infartos lacunares (infartos císticos pequeños con un tamaño menor de $2 \mathrm{~cm}$ que afectan las arterias penetrantes pequeñas paramedianas de un diámetro menor de $0.5 \mathrm{~mm})(1)$. Otro dato importante fue el tamaño del área afectada por el ACV, ya que se encontró que en los pacientes diabéticos son mas frecuentes los accidentes cerebrovasculares que afectan mas del $30 \%$ del parénquima de ambos hemisferios cerebrales.

La asociación entre diabetes y ACV ocurre a través de diversos factores, reconociéndose una asociación independiente $(1,7,8)$. Existen factores demográficos que aumentan el riesgo de $\mathrm{ACV}$ en la población diabética; la edad es el factor de riesgo más importante tanto en la población diabética como en la no-diabética; la raza es otro factor de riesgo siendo mayor la prevalencia de $\mathrm{ACV}$ en los pacientes diabéticos de raza negra y amarilla (9). Asimismo, se sabe que las mujeres diabéticas pierden los factores de protección propios de su sexo por lo que la prevalencia de ACV es similar en los pacientes diabéticos hombres o mujeres (1). En el presente estudio se encontró que los pacientes diabéticos y no diabéticos tuvieron la misma edad promedio de 
ocurrencia de ACV. En cuanto a la influencia del género de la población diabética, no se encuentra preponderancia de ningún género y la relación hombre/ mujer fue de 1, sin embargo, se encontró que los diabéticos hombres fueron más jóvenes que las mujeres diabéticas, lo que nos indicaría que las mujeres diabéticas conservan los factores protectores que retrasan la ocurrencia de ACV en ellas a pesar de la diabetes. Finalmente, la raza mas afectada fue la mestiza, tanto en diabéticos como no diabéticos.

La diabetes es una enfermedad que puede cursar asintomática y aún así se constituye en un importante factor de riesgo para la ocurrencia de $\mathrm{ACV}$ o de infarto agudo de miocardio (10).

Existe una gran proporción de población con diabetes no diagnosticada que tienen un riesgo elevado de presentar eventos tromboembólicos, como por ejemplo, los pacientes que presentan hiperglicemia sin historia de diabetes $(11,12)$. Por esto, es importante que en nuestro estudio en el 27\% de los pacientes diabéticos con ACV, el diagnóstico de diabetes se realizara durante la ocurrencia del ACV; esta información se aclara aún más al encontrar que este grupo presentaba una alta frecuencia de otras patologías asociadas a la ocurrencia de ACV como la hipertensión arterial y la nefropatía diabética que como se sabe acompañan a una diabetes de larga data.

Existe una relación directa entre el tiempo de enfermedad de diabetes y la ocurrencia de ACV. La duración de la diabetes es un factor importante que contribuye a aumentar el riesgo de ACV (13), pues hay mas tiempo para que ocurra daño de los pequeños y largos vasos cerebrales intracraneales, enfermedad ateroesclerótica cardiovascular, vascular periférica y cerebrovascular $(14,15)$. El daño tisular producto de la hiperglicemia crónica se da por la glicación de las proteínas tisulares y otras macromoléculas y una excesiva producción de compuestos de poliol a partir de la glucosa (15). Por otro lado, los altos niveles de insulina son un factor de riesgo de ateroesclerosis, el endotelio microvascular es más susceptible a los efectos metabólicos y mitogénicos de la insulina que el endotelio de los grandes vasos (16). Los resultados del gráfico $\mathrm{N}^{\circ} 2$ sobre la ocurrencia de ACV en función del tiempo de enfermedad de diabetes sugieren que la diabetes en nuestro grupo estudiado ha jugado un rol preponderante en la ocurrencia del ACV. Esto además se corrobora con el tipo de ACV que se ha observado predominantemente en los pacientes diabéticos. Sin embargo, esta tendencia también se ve en otras complicaciones crónicas que implican compromiso vascular y esto puede cuestionar el rol preponderante de la diabetes. Nuestro estudio nos permite concluir que la diabetes es un factor de riesgo importante para la ocurrencia de ACV, sin embargo el diseño de este estudio no nos permite concluir si la diabetes es un factor de riesgo independiente para la ocurrencia de ACV.

Las complicaciones específicas de la diabetes son la retinopatía, la nefropatía y la neuropatía y los pacientes portadores de cualquier forma de diabetes con suficiente duración, son vulnerables a cualquiera de estas complicaciones, siendo su diagnóstico importante porque su presentación se acompaña de un aumento sustancial de la enfermedad ateroesclerótica de grandes vasos como la enfermedad cerebrovascular. Se sabe que la historia natural de la nefropatía comienza con la presentación de microalbuminuria que puede ocurrir a los cinco años de inicio de la diabetes, luego se presenta la proteinuria y finalmente la insuficiencia renal crónica (5). Los niveles aumentados de albuminuria y proteinuria están asociados con la presentación de enfermedad ateroesclerótica, de tal manera que la incidencia de ACV está relacionada directamente con los niveles de proteinuria (17). Esto se demuestra en este estudio al encontrarse un número significativo de pacientes diabéticos con ACV que eran portadores de nefropatía diabética, encontrándose incluso en pacientes con diagnóstico de insuficiencia renal crónica. También es importante tener en cuenta que el $46 \%$ de pacientes diabéticos con ACV presentaban retinopatía diabética, lo cual nos indica el significativo daño vascular que originó la diabetes, pues los cambios que se aprecian en los vasos retinianos pueden ser similares a los que afectan los vasos cerebrales (10). Otras complicaciones como el pie diabético y la neuropatía diabética se presentaron en menor proporción en nuestros pacientes. Los pacientes que no presentaron complicaciones a largo plazo pertenecían en su mayoría al grupo de pacientes que se les diagnosticó diabetes durante la hospitalización.

En nuestro estudio, la población general con ACV presentó una alta prevalencia de hipertensión arterial previamente diagnosticada (53\%), siendo este antecedente mas frecuente en los diabéticos que en los no diabéticos. Es frecuente que el paciente diabético curse con hipertensión arterial, lo que aumenta el riesgo de ACV. La presión arterial es el factor de riesgo más importante para el ACV $(1,18)$, hay una relación directa entre la ocurrencia de ACV con niveles de presión arterial diastólica mayores de $114 \mathrm{mmHg}$ y con la presencia de hipertrofia ventricular. En este estudio no se pudo cuantificar los niveles de presión arterial antes de ocurrir el $\mathrm{ACV}$, pero se encontró que un número significativo de pacientes era portador de hipertrofia ventricular izquierda. 
La dislipidemia es un factor de riesgo importante para la ocurrencia de ACV (19), de tal manera que niveles altos de colesterol total (>200 mg/dl), bajos niveles de colesterol HDL ( $<40 \mathrm{mg} / \mathrm{dl}$ ), niveles altos de colesterol $\mathrm{LDL}(>130 \mathrm{mg} / \mathrm{dl}$ ) y niveles altos de triglicéridos (>150 $\mathrm{mg} / \mathrm{dl}$ ) aumentan de tres a cinco veces el riesgo de ACV en pacientes diabéticos $(19,20,21)$. En la población estudiada, se observó una alta proporción de pacientes que presentaban estas alteraciones, encontrándose que es mas frecuente que los diabéticos presenten niveles de colesterol total mayor de $200 \mathrm{mg} / \mathrm{dl} \mathrm{y} \mathrm{colesterol} \mathrm{LDL}$ mayor de $130 \mathrm{mg} / \mathrm{dl}$. También se ha señalado que hay una asociación negativa independiente entre niveles bajos de colesterol HDL y la ocurrencia de ACV tipo isquémico $(22,23)$, esto se evidencia en nuestro estudio en el que se encuentra una alta proporción de pacientes diabéticos que tienen niveles de colesterol HDL menores de $40 \mathrm{mg} / \mathrm{dl}$. Debe anotarse que es muy importante un control más estricto del perfil lipídico y que todo paciente que es hospitalizado por ACV debe tener un control sanguíneo de su perfil lipídico.

Otro factor de riesgo importante para la ocurrencia de ACV en pacientes diabéticos es el antecedente de ACV previo (19), esto coincide con las características de la población del presente estudio al observarse que este antecedente era mas frecuente en los pacientes diabéticos $(27 \%)$ que en los no diabéticos $(15 \%)$.

La información obtenida para la realización del presente estudio no permite obtener conclusiones válidas sobre otros factores de riesgo para ACV relacionados con el estilo de vida de los pacientes como por ejemplo, el tabaco, el alcohol, uso de anticonceptivos orales, obesidad, esto por la carencia de esta información en las historias clínicas.

Diversos estudios han demostrado un aumento en la morbimortalidad de los pacientes diabéticos que sufren ACV. En nuestro estudio no se encontraron diferencias entre pacientes diabéticos y no diabéticos en el tiempo de hospitalización. En cuanto a las complicaciones que se presentaron durante la hospitalización, la patología cardiovascular fue la mas frecuente en la población general y al mismo tiempo, fue mas frecuente en los pacientes diabéticos que en los no diabéticos. La infección del tracto urinario también se presentó con mas frecuencia en los pacientes diabéticos que en los no diabéticos.

Es importante también la observación que ningún paciente diabético falleció durante su hospitalización por ACV, pero podría tener relación al periodo de estudio y al número de casos estudiados. Los pacientes de nuestra población que fallecieron fueron pacientes no diabéticos que tuvieron ACV tipo hemorrágico. La explicación es que los pacientes diabéticos presentaron en su mayoría $\mathrm{ACV}$ tipo isquémico y que el ACV hemorrágico que es el que produce mayor mortalidad por la presencia de eventos secundarios como el edema cerebral.

No puede olvidarse que el impacto social y emocional de la diabetes, sus complicaciones tardías y las demandas de la terapia originan una importante presión psicosocial en los pacientes y sus familias (15). Es importante observar que la población que presentó $\mathrm{ACV}$ que tenía diagnóstico previo de diabetes no llevaba un adecuado control de su enfermedad al no tomar su medicación de manera regular o al no modificar su estilo de vida.

Los resultados del presente estudio indican que el paciente diabético debe ser manejado de manera integral, tanto en el control de la glicemia como en el manejo de los otros factores de riesgo asociados a la ocurrencia de ACV.

\section{Correspondencia:}

Elsa Rosa Neira Sánchez

Av. La Molina Mz 3LL - L 12 Sol de la Molina

Lima - Perú

\section{BIBLIOGRAFIA}

1. Bell D. Stroke in the diabetic patient. Diabetes Care 1994; 17:213-219.

2. Burchfiel CM, Curb JD, Rodriguez BL, et al. Glucose intolerance and 22-year stroke incidence: The Honolulu Heart Program. Stroke 1994; 25:951-957.

3. Manson JE, Colditz GA, Stampfer HL et al. A prospective study of maturity-onset diabetes mellitus and risk of coronary heart disease and stroke in women. Arch Intern Med 1991; 151:1141-1147.

4. Mankovsky BN, Metzger BE, Molitch ME, Biller J. Cerebrovaseular disorders in patients with diabetes mellitus. J Diabetes Complications 1996; 10:228-242.

5. Nathan DM. Long-term complications of diabetes mellitus. N Engl J Med 1993; 328:1676-1685.

6. Bonita R. Epidemiology of stroke. The Lancet 1992; 330:342-372.

7. Bronner LL, Kanter DS, Manson JE. Primary prevention of stroke. N Engl J Med 1995; 333:1392-1400.

8. Pullicino PM, Xuereb M, Aquilina J, Piedmonte MR. Stroke following acute Myocardial infarction in diabetics. J Intern Med 1992; 231:287-293.

9. Kittner SJ, White LR, Losonczy KG ct al. Black-white differences in stroke incidence in a national sample: the contribution of hypertension and diabetes mellitus. JAMA 1990; 264:1267-270.

10.Asplund K, Hgag E, Helmers C, et al. The natural history 
of stroke in diabetic patients. Acta Med Scand 1980; 207:417-424.

11.Horan MI. Diabetes and hypertension. En: Diabetes in America data complied 1984. Harris MI, Hamman RF, eds. NIH publication No. 85-1468; Bethesda, National Institutes of Health, 1985: 1-22.

12.Abbot RD, Donahue RP, MacMahon SW, et al. Diabetes and the risk of stroke: The Honolulu heart program. JAMA 1987; 257:949-952.

13.Tuomilehto J, Rastenyt AD, Jousilahti P, et al. Diabetes mellitus as a risk factor for death from stroke. Prospective study of the middle-aged Fnnish population. Stroke 1996; 27:210-215.

14.Caplan LR. Diabetes and brain ischemia. Diabetes 1996; Suppl 3:S95-97

15.Report of the Expert Committee on the Diagnosis and Classification of Diabetes Mellitus.Diabetes Care 1997; 20:1183-1197.

16.Zunker P, Schick A, Buschmann HC, et al. Hyperinsulinism and cerebral microangiopathy. Stroke 1996; 27:219-223.

17.Miettinen H, Haffner SM, Lehto S, et al. Proteinuria pre- dicts stroke and other atheroesclerotic vascular disease events in non-diabetic and non-insulin-dependent diabetic subjects. Stroke 1996; 27:2033-2039.

18.Roehmholdt ME, Palumbo PJ, Whisnant JP, et al. Transient ischemic attack and stroke in a community-based diabetic cohort. Mayo Clin Proc 1983; 58:56-58.

19.Lehto S, Ronnemaa T, Pyorala K, Laakso M. Predictors of stroke in middle-aged patients with noninsulinadependen diabetes. Stroke 1996; 27:63-68.

20.Hachinski V, Graffagnino C, Beaudry M, et al. Lipids and stroke: a paradox resolved. Arch Neurol 1996; 53:303-308.

21.Dyker AG, Weir CJ, Lees KR. Influence of cholesterol on survival after stroke: retrospective study. BMJ 1997; 314:584-1588.

22.Clark CM, Lee DA. Prevention and treatment of the complications of diabetes mellitus. N Engl J Med 1995; 332:1210-1217.

23.TaMe D, Yaari S, Goldbourt U. High-density lipoprotein cholesterol and risk of ischaemic stroke mortality. A 21year follow-up of 8586 men from the israeli Ischemic Heart Disease Study. Stroke 1997; 28:83-87. 